International Journal of Engineering \& Technology, $7(4.4)(2018) 7-8$
International Journal of Engineering \& Technology
SPC
Website: www.sciencepubco.com/index.php/IJET
Research paper

\title{
Iterative Approach for Performance Improvement of PLC Systems
}

\author{
Yu Min Hwang, Young Ghyu Sun, Issac Sim and Jin Young Kim* \\ ${ }^{1}$ Department of Wireless Communications Engineering, Kwangwoon University, Seoul 01897, Korea.
}

\begin{abstract}
In this paper, we propose Turbo equalizer scheme using zero-forcing (ZF) and simulate in power line communication (PLC) systems with high speed. Due to impulsive noise and multipath fading in PLC systems, inter-symbol interference (ISI) happens. We employ Turbo equalizer scheme to get over ISI and improve performance. From simulation results, it is confirmed that the proposed Turbo equalizer can remarkably improve PLC system performance.
\end{abstract}

Keywords:Power line communication, multi-path fading, turbo equalizer.

\section{Introduction}

Smart grid has been an important issue not only for electric industry but also for the industry overall such as car, power distribution, computer, communication, and so on. The smart grid provides a variety of services such as home area network (HAN), supervisory control and data acquisition (SCADA), energy management service (EMS) and distribution automation system (DAS) by converging with IT techniques [1-2].

Behind above advantages, there are several challenges should be solved. One of them is impulse noise, which is caused from random on/off of electrical devices. Its power spectral density (PSD) can be as much as $50 \mathrm{~dB}$ higher than the background noise spectrum. Therefore, it can erase data symbol blocks while data is transmitted [3].

In this paper, to compensate the channel effects and mitigate impulsive noise in power line communications (PLCs), we employ Turbo equalizer which is stronger than other linear equalizer schemes.

\section{Channel Model for PLCs}

The power line medium is an unstable transmission channel owing to the variance of impedance caused by the variety of appliances that could be connected to the power outlets. The impedance is mainly influenced by the characteristic impedance of the cables, the topology of the considered part of network and the nature of the connected electrical loads. M. Zimmermann and K. Dostert [4] proposed a multipath scenario with frequency selective fading and the scenario is considered in this paper. The delay $\tau_{-} i$ of a path and the channel model is referred to [4-5].

\section{Turbo Equalizer}

We describe Turbo equalization and its process. When binary data is concerned, such as the bits $\alpha_{k}$, it is convenient to work with log-likelihoodratios (LLRs) rather than probabilities. The LLR $L(a)$ including the same information as any of the two a prioriprobabilities for $P(a=1)$ or $P(a=0)$ forbinary variablea given $\mathbf{y}$ is defined as:

$$
L(a \mid y)=\ln \frac{P(a=1 \mid y)}{P(a=0 \mid y)}
$$

Thus, the decision rule can be applied as:

$\hat{a}_{k}= \begin{cases}1, & L\left(a_{k} \mid \mathbf{y}\right) \geq 0, \\ 0, & L\left(a_{k} \mid \mathbf{y}\right)<0 .\end{cases}$

From Bayes rule, the equalizer outputs a posteriori LLR defined as:

$$
\Lambda\left(c_{k} \mid \mathbf{y}\right)=\ln \frac{\sum_{\forall \mathbf{c}: c_{k}=+1} p(\mathbf{y} \mid \mathbf{c}) \prod_{i=1}^{K} \mathrm{P}\left(c_{i}\right)}{\sum_{\forall \mathbf{c}: c_{k}=-1} p(\mathbf{y} \mid \mathbf{c}) \prod_{i=1}^{K} \mathrm{P}\left(c_{i}\right)}=L_{e x t}\left(c_{k} \mid \mathbf{y}\right)+L\left(c_{k}\right)
$$

where $P\left(c_{k}=+1 \mid \mathbf{y}\right)$ and $P\left(c_{k}=-1 \mid \mathbf{y}\right)$ are probabilities that transmitted signal $c_{k}$ is +1 or -1 when $\mathbf{y}$ is received, respectively. Further, $\forall \mathbf{c}: c_{k}=+1$ means the case that $k^{t h}$ element of arbitrary $\mathbf{c}$ is ' +1 '. $P(\mathbf{y} \mid \mathbf{c})$ is joint probability density function (PDF) for received signal $y$ when the transmitted signal is $\mathbf{c} . P\left(c_{i}\right)$ is probability for $k^{\text {th }}$ element of $\mathbf{c} . \Lambda\left(c_{k} \mid \mathbf{y}\right)$ is consisted of two elements, so-called the extrinsic information $L_{\text {ext }}\left(c_{k} \mid \mathbf{y}\right)$ and the intrinsic (apriori) information $L\left(c_{k}\right) . \Lambda\left(c_{k} \mid \mathbf{y}\right)$ can be transformed to probabilities as below:

$$
P\left(c_{k}=c \mid \mathbf{y}\right)=\frac{\exp \left(-c \cdot \Lambda\left(c_{k} \mid \mathbf{y}\right)\right)}{1+\exp \left(-\Lambda\left(c_{k} \mid \mathbf{y}\right)\right)}
$$


where $c \in\{0,1\}$. After de-interleaving $P\left(c_{k} \mid \mathbf{y}\right)$ to $P\left(b_{k} \mid \mathbf{y}\right)$, we confront the classical problem of decoding a binary convolutional code with probabilistic input.

\section{Simulation Results}

Simulation is carried out in the number of power line branches is five. The length of branches is set up randomly in the range of $2 \mathrm{~m}$ to $10 \mathrm{~m}$ every packet. And it is $60 \mathrm{~m}$ that the length of main line. The state of each device at the end of branch is randomly on or off. Last, BPSK modulation formats are used as modulation method. We analyzed performance with regard to bit error rate (BER).

In the case using ZF only, The result shows that BER performance is about $14 \mathrm{~dB}$ at $10^{-2}$ point and $24 \mathrm{~dB}$ at $10^{-3}$ point, whereas, in the case of ZF with Turbo equalizer, it shows about $11 \mathrm{~dB}$ and $16.5 \mathrm{~dB}$ at BER of $10^{-2}$ and $10^{-3}$, respectively. As signal-to-noise ratio (SNR) is getting lager, performance improvement is bigger. Also, the performance is improved as the number of iteration for Turbo equalization increases, but amount of performance improvement is smaller.

\section{Conclusion}

In this paper, we proposed and simulated Turbo equalizer scheme using ZF for high speed power line communication systems in order to overcome the effect of ISI caused by impulsive noise and multipath fading. From simulation results, it is confirmed that performance of the proposed system can be remarkably improved when the Turbo equalizer applied.

\section{Acknowledgment}

This work was supported by the Basic Science Research Program through the National Research Foundation of Korea funded by the Ministry of Education under Grant NRF-2016R1D1A1B03933872.

\section{References}

[1] J. Y. Kim, Power Line Communication Systems, GS Intervision Publisher, Seoul, Korea, 2009

[2] Francesco Chiti, Romano Fantacci, Dania Marabissi and Andrea Tani, "Performance evaluation of an efficient and reliable multicast power line communication system", IEEE J. Sel. Areas Commun., vol. 34, no. 7, July 2016.

[3] P. Degauque et al., "Power-line communication: Channel characterization and modeling for transportation systems," IEEE Veh. Technol. Mag., vol. 10, no. 2, pp. 28-37, June 2015

[4] M. Zimmermann and K. Dostert, "Analysis and modeling of impulsive noise in broad-band powerline communications," IEEE Trans. Electromagnetic Compatibility, vol. 44, no. 1, pp. 249-258, Feb. 2002.

[5] L. Lampe, A. M. Tonello and T. G. Swart, Power line communications: Principles standards and applications from multimedia to smart grid, Chichester, U.K.:Wiley, 2016. 